\title{
Selective activation of TRPA1 ion channel by skin sensitizer nitrobenzene DNFB
}

\author{
Han $\mathrm{Wu}^{1}$, Yaxuan $\mathrm{Qu}^{1}$, Xiaoying $\mathrm{Sun}^{1}$, and Kewei Wang ${ }^{1}$ \\ ${ }^{1}$ Qingdao University Medical College
}

June 15,2021

\begin{abstract}
BACKGROUND AND PURPOSE: Chemical 2, 4-dinitrofluorobenzene (DNFB), commonly called as Sanger's reagent, is well known as skin sensitizer to cause dermatitis. However, how the DNFB causes skin inflammation remains unknown. In this study we aimed at identifying the molecular target that DNFB acts on. EXPERIMENTAL APPROACH: We used a fluorescent calcium imaging plate reader as an initial screening assay and patch-clamp recordings for validation. Molecular docking in combination with site-directed mutagenesis was carried out to investigate DNFB binding sites in TRPA1 ion channel. KEY RESULTS: We found the chemical DNFB that selectively activates TRPA1 channel with EC50 of $2.36 \pm 0.26 \mu \mathrm{M}$. Single-channel recording reveals that DNFB increases the channel open probability and acts on three residues C621, Y658 and E625 critical for DNFB-mediated TRPA1 activation. CONCLUSION AND IMPLICATIONS: Our findings not only explain a molecular mechanism underlying the dermatitis and pruritus caused by chemical DNFB, but also provides a molecular tool that is 7.5time more potent than current AITC molecule and can be used for elucidating TRPA1 channel pharmacology and pathology.
\end{abstract}

\section{Selective activation of TRPA1 ion channel by skin sensitizer nitrobenzene DNFB}

Han $\mathrm{Wu}^{1, \S}$, Yaxuan $\mathrm{Qu}^{1, \S}$, Xiaoying Sun ${ }^{1,2, \mathbb{I}}$ and KeWei Wang ${ }^{1,2, \mathbb{I}}$

${ }^{1}$ Department of Pharmacology, School of Pharmacy, Qingdao University, Qingdao 266021, China; ${ }^{2}$ Institue of Innovative Drugs, Qingdao University, Qingdao 266021, China;

$\S$ These two authors contributed equally to this paper.

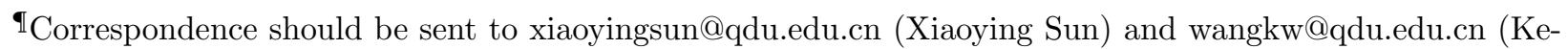
Wei Wang)

\section{Running title: Chemcial DNFB activates TRPA1}

Data Availability Statement : The data that support the findings of this study are available from the corresponding author upon reasonable request. Some data may not be made available because of privacy.

\section{Bullet point summary}

\section{What is already known}

Chemical nitrobenzene DNFB, also known as Sanger's reagent and skin sensitizer, causes dermatitis.

Activation of TRPA1 ion channel is involved in allergic contact dermatitis.

What this study adds:

DNFB selectively activates TRPA1 current and increases single channel open probability. 
Three residues in TRPA1 irritant sensing domain are critical for DNFB binding.

\section{Clinical significance:}

Pharmacological inhibition of TRPA1 may hold promise for potential therapy of dermatitis and pruritus.

BACKGROUND AND PURPOSE:Chemical 2, 4-dinitrofluorobenzene (DNFB), commonly called as Sanger's reagent, is well known as skin sensitizer to cause dermatitis. However, how the DNFB causes skin inflammation remains unknown. In this study we aimed at identifying the molecular target that DNFB acts on.

EXPERIMENTAL APPROACH: We used a fluorescent calcium imaging plate reader as an initial screening assay and patch-clamp recordings for validation. Molecular docking in combination with sitedirected mutagenesis was carried out to investigate DNFB binding sites in TRPA1 ion channel.

KEY RESULTS: We found the chemical DNFB that selectively activates TRPA1 channel with $\mathrm{EC}_{50}$ of $2.36 \pm 0.26 \mu \mathrm{M}$. Single-channel recording reveals that DNFB increases the channel open probability and acts on three residues C621, Y658 and E625 critical for DNFB-mediated TRPA1 activation.

CONCLUSION AND IMPLICATIONS: Our findings not only explain a molecular mechanism underlying the dermatitis and pruritus caused by chemical DNFB, but also provides a molecular tool that is 7.5-time more potent than current AITC molecule and can be used for elucidating TRPA1 channel pharmacology and pathology.

\section{Abbreviations:}

2-APB, 2-aminoethoxydiphenyl borate; A-96, A-967079; ACD, allergic contact dermatitis; AD, atopic dermatitis; AITC, allyl isothiocyanate; ANOVA, one-way analysis of variance; CA, cinnamaldehyde; Cap, capsaicin; cryo-EM, cryoelectron microscopy; DMEM, Dulbecco's modified Eagle's medium; DNCB, 2, 4dinitrochlorobenzene; DNFB, 2, 4-dinitrofluorobenzene; FBS, fetal bovine serum; GFP, Green fluorescent protein; GSK101, GSK1016790A; HBSS, Hanks' balanced salt solution; HEK293, human embryonic kidney 293; IL-1, interleukin-1; MO, mustard oil; PDB, Protein Data Bank; PGE2, Prostaglandin E2; RFU, relative fluorescence unit; RR, ruthenium red; thermo-TRPs, Thermal transient receptor potentials; TRP, transient receptor potential; TRPA1, Transient receptor potential Ankyrin1; TRPV, TRP vanilloid.

Key words: TRPA1; Nitrobenzene; DNFB; skin sensitizer; contact dermatitis; electrophiles; TRPs

\section{Introduction}

Chemical nitrobenzene compounds such as 2, 4-dinitrofluorobenzene (DNFB) and 2, 4-dinitrochlorobenzene (DNCB) have long been known to cause contact skin irritation and sensitization and they are also used for establishment of skin inflammatory models in rodents, (Landsteiner and Chase, 1941; Ren et al., 2019; Tingle et al., 1990). Topical application of DNFB, commonly called Sanger's reagent used for protein sequencing, mediates contact hypersensitivity and induces allergic contact dermatitis (ACD) (Garcia-Perez, 1978; Hsieh et al., 1996). DNFB is also commonly used to sensitize skin and cause colitis in mice (Rijnierse et al., 2006a; Rijnierse et al., 2006b). Chemical DNFB as an allergen also elicits immune reactions by inducing mast cell degranulation and releases of histamine (Ren et al., 2019), interleukin-1 (IL-1) and prostaglandin E2 (PGE2) (Natsuaki et al., 1989). However, how DNFB causes skin sensitization and allergic contact dermatitis at molecular level remains unknown.

A growing number of evidences have indicated that activation of transient receptor potential ankryn 1 (TRPA1) ion channels is involved in skin inflammation (Tóth. et al., 2014). TRPA1 is robustly expressed in primary sensory nerve terminals (Mandadi and Roufogalis, 2008) and in numerous non-neuronal cell types of the skin (Atoyan et al., 2009) and CD4+ T lymphocytes that play a central role in the adaptive immune response (Bertin et al., 2017; Li et al., 2020). Activation of TRPA1 by icilin in keratinocytes leads to an eluviation of proinflammatory cytokine interleukin-1 (IL-1), which suggests a role of TRPA1 in promoting cutaneous inflammation (Atoyan et al., 2009). Pharmacological inhibition or deficiency of TRPA1 alleviates 
inflammation of atopic dermatitis (AD) (Oh et al., 2013; Zeng et al., 2021). Conversely, specific activation of TRPA1 by agonist mustard oil (MO) leads to a severe colitis, which is inhibited by HC-030031 or reduced in TRPA1-/-mice (Engel et al., 2011).

TRPA1 is a temperature-sensitive and calcium-permeable cation channel with a four-fold symmetry around the central ion conductance pathway and proximal cytoplasmic regions involved in electrophile detection (Liao et al., 2013; Paulsen et al., 2015; Story et al., 2003). TRPA1 is a sensor for chemical irritants, such as MO (Jordt et al., 2004), acrolein, cinnamaldehyde (CA) (Bandell et al., 2004) and allyl isothiocyanate (AITC) (Sandor et al., 2016). TRPA1 is also activated by harmful electrophiles that are recognized by the channel via covalent modifications of specific cysteine residues located in the cytoplasmic domain (Suo et al., 2020). All these investigations suggest us that skin sensitizer DNFB might act on TRPA1 channel that is considered a key player in skin inflammation.

In this study, we discovered that chemical DNFB that specifically activates TRPA1 channels through its binding to three key residues critical in the channel coupling domain for electrophile irritant sensing using assays of patch clamp recordings in combination with site-directed mutagenesis and molecular docking. Our findings not only help explain the mechanistic insights into nitrobenzene DNFB-induced contact dermatitis, but also provide a molecular tool for further understanding TRPA1 channel pharmacology in skin-related diseases.

\section{Materials and methods}

Reagents and compounds

Compounds DNFB (MW: 516.45), 2-aminoethoxydiphenyl borate (2-APB), capsaicin (Cap), ruthenium red (RR), GSK1016790A (GSK101), A-967079 (A-96) and allyl isothiocyanate (AITC) were purchased from Sigma-Aldrich (St. Louis, MO). RR were made as stock solutions in before use internal solution. Other compounds were made as stock solutions in DMSO before use. Compounds used for the measurement of intracellular fluorescent calcium were diluted in the Hanks' balanced salt solution (HBSS). Compounds were diluted in perfusion solution for patch-clamp recordings. DNFB was dissolved in solvent (acetone/olive oil $=4: 1$ ) for establishment of DNFB induced inflammation model (Bhol and Schechter, 2005).

Animals

The male C57BL/6 mice (aged 6-8 weeks, weighing $20 \pm 2 \mathrm{~g}$ ) mice we buy are from the Vital River Laboratory Animal Technology Co. Ltd. (Beijing, China). In order to adapt them to the new environment, the mice were raised at least a week in the case of sufficient supply and free intake and has a normal circadian rhythm before the experiment. All animal tests were authorized by the Institutional Animal Care and Use Committee of Qingdao University Health Science Center and were performed in accordance with institutional and national guide-lines for the use and care of animals for experiments.

Establishment of DNFB induced inflammation model of the dorsal skin in mice

The hair of mice was shaved firstly, and then the remaining hair was removed with depilatory cream. The interval of one day is recorded as the day 0 and is not processed. At day $1,100 \mu \mathrm{l} 0.5 \%$ DNFB was topically applied to sensitize the prepared dorsal skin of mice. No DNFB treatment at day 2, 3 and 4 . At day 5 and day $6,50 \mu \mathrm{l} 0.2 \%$ DNFB was applied to challenge the skin respectively. The solvent (acetone/olive oil, 4:1) was used as a control.

\section{Histology}

We obtained the dorsal skin tissue of the mice after taking pictures at day 7 . The obtained tissues were fixed overnight in $4 \%$ formaldehyde at 4 , dehydrated in ethanol, embedded in paraffin, and cut into 6 $\mu \mathrm{m}$-thick sections for H\&E staining. Skin paraffin sections were baked at 60 for one hour before staining. The sliced sections were imaged with a bright-field microscope (Eclipse Ti-S; Nikon) with a charge-coupled device camera (DS-Ri2; Nikon). 
Cell culture and transfection

The human embryonic kidney cells (HEK293) were cultured in the Dulbecco's minimal essential medium with supplement of $10 \%$ of fetal bovine serum (FBS) at 37 with $5 \% \mathrm{CO}^{2}$. HEK293 cells were seeded in a 6-well plate for intracellular calcium measurement. For whole-cell patch clamp recordings, cells were cultured on glass coverslips. The HEK293 cells were transiently cotransfected in $350-\mathrm{mm}$ petri dishes or a $350-\mathrm{mm}$ well on a 6 -well plate with $1600 \mathrm{ng}$ of cDNA plasmid encoding wild-type or mutant human TRPA1 and and other TRP channels, using Lipofectamine 2000 (Invitrogen). The cells were used after 24-48 h transfection. Human TRPA1 plasmids (Gene ID: 8989) including wild-type or mutants were verified by DNA sequencing.

Measurement of intracellular calcium in fluorescent imaging plate reader FlexStation 3 assay

The Cal-520TM PBX Calcium Assay Kit (AAT Bioquest, Sunnyvale, CA) was used to detect the change of intracellular calcium in a population of cells with Multi-Mode Microplate Reader in FlexStation 3 assay (Molecular Devices, San Jose, CA). HEK293 cells were transiently transfected with cDNA of TRP channels before seeded at a density of $\sim 40,000$ cells per well in a 96-well black-walled plate (Thermo Fisher Scientific, Waltham, MA) for an overnight culture at 37 with $5 \% \mathrm{CO}^{2}$. Cells were incubated with the calcium fluorescent dye provided in the Cal-520TM PBX Calcium Assay Kit at $37 \mathrm{for} 1.5 \mathrm{~h}$, and the values of relative fluorescence unit (RFU) were measured by FlexStation3 under the wavelength at $485 \mathrm{~nm}$ (excitation) and $515 \mathrm{~nm}$ (emission) with an interval of $1.6 \mathrm{~s}$ (Sun et al., 2017).

Patch clamp recordings

All patch-clamp recordings in two different configurations of macroscopic whole-cell and microscopic insideout patch clamp recordings were conducted after 12-24 h transfection with an EPC10 amplifier powered by PatchMaster software (HEKA) at room temperature (23-25). The borosilicate glass pipettes were pulled using a DMZ universal electrode puller (Zeitz-Instruments $\mathrm{GmbH}$ ) with the resistances of 3-5 M $\Omega$ (whole cell) or 5-7 M $\Omega$ (inside-out) when filled with a solution containing (in mM): (1) $150 \mathrm{NaCl}, 2 \mathrm{EGTA}, 10$ HEPES, 1 InsP6 (pH 7.2, adjusted with $\mathrm{NaOH}$ ) for humanTRPA1 (inside-out); (2) $130 \mathrm{NaCl}, 0.2$ EGTA, 3 HEPES (pH 7.2, adjusted with $\mathrm{NaOH}$ ) for others. External solution was prepared ditto to internal solution. Currents were recorded at a potential ramp from $-100 \mathrm{mV}$ to $+100 \mathrm{mV}$ (whole-cell) or a potential of $+80 \mathrm{mV}$ (inside-out).

Molecular docking

The cryo-EM structures of hTRPA1 (Protein Data Bank (PDB) code: 3J9P) were selected as the docking model. The binding pocket were determined according to the reported TRPA1 agonist binding site (Suo et al., 2020) and the Glide method. And the conformation was conducted according to the result score using Rosetta software with default parameters. Binding poses of DNFB and DNCB with the top ten scores were considered as the possible binding modes with hTRPA1.

Site-directed Mutagenesis

The Mut Express II rapid mutagenization kit was used to generate site-directed mutations in TRPA1 (Gene ID: 8989). All the mutants were verified by autosequencing to ensure the correct generation of mutations.

Data analysis

All data are expressed with the mean \pm SEM. Statistical significance was calculated by one-way analysis of variance (ANOVA) and unpaired $t$-test using GraphPad Prism 8.0 and Origin 9 software. A value of $P<$ 0.05 is considered to be statistically significant.

\section{Results}

Skin inflammation induced by topical applications of DNFB in mice. 
DNFB, a known chemical contact sensitizer, is widely used to induce skin inflammation model. We started recapitulating the mouse chronic model of contact dermatitis by topical applications of DNFB. As shown in Fig. 1a, DNFB was topically applied to mouse dorsal skin for three times starting day 1 (100 $\mu$ l of $0.5 \%$ DNFB), day 5 (50 $\mu \mathrm{l}$ of $0.2 \%$ DNFB) and day 6 (50 $\mu \mathrm{l}$ of $0.2 \%$ DNFB). Obvious inflammation of dorsal skin started to develop at day 2 after first application of topical DNFB (Fig. 1b) and aggravated with increase of skin keratinization following subsequent applications of DNFB in time-dependent manner (Fig. 1b). H\&E staining of skin tissue sections further revealed a significant increase of skin thickness and infiltration of inflammatory cells (Fig. 1c). These results indicate that topical applications of DNFB can cause contact dermatitis in mice.

Selective activation of TRPA1 channels by chemical DNFB in calcium fluorescent assay and patch-clamp recordings

To test the effect of DNFB (Fig. 2a) on TRPA1 and other thermo-TRPs, we started using the calcium fluorescent imaging of HEK293 cells expressing several TRP channels in FlexStation3 microplate reader assay. Adding different concentrations $(3-300 \mu \mathrm{M})$ of DNFB caused a dose-dependent increase of intracellular $\mathrm{Ca}^{2+}$ level, as compared with TRPA1 agonist AITC $(300 \mu \mathrm{M})$ as positive control (Fig. 2a). There was a lack of detectable signals from TRPV1 (Fig. 2b), TRPV3 (Fig. 2c) and TRPV4 (Fig. 2d) channels in response to DNFB in the same range of concentration $(3-300 \mu \mathrm{M})$. These results suggest that DNFB selectively activates TRPA1 over the other tested members of TRP channels.

To confirm the activation of TRPA1 channels by DNFB, we recorded the whole-cell currents of TRPA1, TRPV1, TRPV3 and TRPV4 channels expressed in HEK293 cells in the presence of $5 \mu \mathrm{M}$ DNFB. As shown in Fig. 3, DNFB-mediated activation of TRPA1 current was blocked by the channel blocker ruthenium red (RR) at $20 \mu \mathrm{M}$ (Fig. 3a). In contrast, TRPV1 (Fig. 3b), TRPV3 (Fig. 3c) and TRPV4 (Fig. 3d) channels were not responsive to DNFB $(5 \mu \mathrm{M})$, although these channels were activated by their agonists such as 1 $\mu \mathrm{M}$ capsaicin for TRPV1, $50 \mu \mathrm{M}$ 2-APB for TRPV3 and 0.1 $\mu \mathrm{M}$ GSK101 for TRPV4. Consistent with the data from calcium fluorescent assay, these results confirmed that DNFB is a selective agonist of TRPA1.

To determine the potency of DNFB on TRPA1 activation, we made the whole-cell recordings of TRPA1 currents in the presence of different concentration of DNFB (0.1-100 $\mu \mathrm{M})$ and observed a dose-dependent activation of TRPA1 currents with an $\mathrm{EC}_{50}$ value of $2.36 \pm 0.26 \mu \mathrm{M}$ (Fig. 3e left panel and f). As a control, TRPA1 agonist AITC (3-3000 $\mu \mathrm{M})$ also elicited a dose-dependent activation of the channel current with an $\mathrm{EC}_{50}$ value of $17.77 \pm 5.02 \mu \mathrm{M}$ (Fig. 3e, right panel and f), consistent with a previous report (Cavanaugh et al., 2008). These results indicate that DNFB activates TRPA1 currents in dose-dependent manner with approximately 7.5-fold potency better than AITC.

Direct targeting of single TRPA1 channels by DNFB

To further confirm DNFB directly acting on single TRPA1 channels, we performed the single-channel recordings in an inside-out patch configuration. As a control, application of TRPA1 agonist $300 \mu \mathrm{M}$ AITC increased the channel opening levels to level 2 from level 1 with the single channel conductance about $148.37 \pm 0.38$ $\mathrm{pS}$ and channel open probability about to $25.58 \%$ from $0.59 \%$ of basal control $(n=9-11, P<0.05)$ (Fig. $4 \mathrm{a}, \mathrm{b}$ and c). Adding DNFB at $300 \mu \mathrm{M}$ resulted in a significant increase of the channel opening from level 1 to level 4 with single channel conductance about $153.5 \pm 0.05 \mathrm{pS}$ and channel open probability to $33.25 \%$ $(n=6-14, P<0.001)$ from $0.59 \%$ (Fig. $4 \mathrm{a}, \mathrm{b}$ and $\mathrm{c})$. These results confirmed that DNFB activates TRPA1 currents by directly acting on single channels through increase of channel open probability.

Identification of TRPA1 channel residues critical for DNFB binding

To identify residues critical for DNFB binding to TRPA1, we carried out the molecular docking of DNFB onto the cryo-EM structure of human TRPA1 (PDB: 3J9P) using the Rosetta modeling. As shown in Fig. $5 \mathrm{a}-\mathrm{b}$, the docking reveals that DNFB is confined near the short helixes (H2, H4 and H5) in the coupling domain that is involved in electrophile irritant sensing (Suo et al., 2020). The ortho-nitro group of DNFB are both bound through hydrogen bonds to Cys621 at the end of short helix H2 and Tyr658 in the loop between 
$\beta 1.2$ and H4 (Fig. 5a and b). In particular, in the binding model of DNFB, fluorine atoms unique in DNFB interact with Tyr684 through halogen bond, thus fixing the orientation of para-nitro pointing to $\mathrm{H} 2$, which causes the hydrogen bond between the para-nitro and Glu625, the $\pi$-Alkyl interaction with Ala688 and the $\pi$-Sulfur interaction with Cys621 to maintain conformation and position of the ligand (Fig. 5b). There are also some aromatic amino acids, such as Tyr681, interacting with DNFB through weak van der Waals forces and seems to be inessential for the binding of DNFB to TRPA1.

To confirm that Cys621 and Tyr658 in hTRPA1 are required for the DNFB binding, we introduced glycine and alanine mutations of C621G, Y658A and E681A. As shown in Fig. 5, application of $10 \mu \mathrm{M} \mathrm{DNFB}$ was unable to elicit detectable currents of TRPA1 $1_{\mathrm{C} 621 \mathrm{G}}$ (Fig. $5 \mathrm{~d}$ and $\mathrm{h}$ ) and TRPA1 $1_{\mathrm{Y} 658 \mathrm{~A}}$ (Fig. $5 \mathrm{f}$ and $\mathrm{h}$ ) mutants. In contrast, DNFB at $10 \mu \mathrm{M}$ elicited robust E681A currents (Fig. 5g and h). Interestingly, the 10 $\mu \mathrm{M}$ DNFB cannot evoke the current of the TRPA1 $1_{\mathrm{E} 625 \mathrm{~A}}$, suggesting that Glu625 is essential for activation of TRPA1 by DNFB (Fig. 5e and h).

\section{Discussion}

In this study, we identified an irritant chemical nitrobenzene DNFB that is a well known to cause allergic contact dermatitis and can selectively activate TRPA1 channels. DNFB is about 7.5 times more potent than AITC that is currently available agonist of TRPA1. At the single-channel level, DNFB increases the channel open probability through binding to three key residues C621, Y658 and E625 in the channel coupling domain that functions to sense electrophile irritants (Suo et al., 2020). Our identification of chemical DNFB as a selective TRPA1 agonist not only provides a powerful tool for further understanding of the channel pharmacology and pathology, but also demonstrates the importance of TRPA1 as a potential therapeutic target for allergic contact dermatitis (Oh et al., 2013).

Previous studies have shown that several TRP channels such as TRPA1, TRPV1, TRPV3 and TRPV4 are implicated in skin physiology and pathology including skin inflammation (Luo et al., 2018; Nilius et al., 2007; Southall et al., 2003; Toth et al., 2014; Vay et al., 2012; Yoshioka et al., 2009). Our selectivity evaluations indicate that DNFB specifically actives TRPA1 that is featured of a unique electrophilic sensing pocket located in the channel C-terminal coupling domain (Suo et al., 2020). The electrophilic sensing pocket is highly rich in reactive cysteines such as C621 and C655 (Hinman et al., 2006; Macpherson et al., 2007; Suo et al., 2020; Zhao et al., 2020), and the electrophile sensing region is surrounded by a number of nucleophilic aromatic amino acids for facilitating the entry of electrophiles (Suo et al., 2020). In contrast, the cryoelectron microscopy structures of TRPV1 (Cao et al., 2013), TRPV3 (Singh et al., 2018) and TRPV4 (Deng et al., 2018) reveal that these three channels are lack of the electrophile sensing coupling domain that serves to sense electrophilic irritants (Paulsen et al., 2015), which explains the selective activation of TRPA1 by chemical DNFB (Saarnilehto et al., 2014).

Chemical DNFB as an electrophilic reagent binds to TRPA1 through non-covalent hydrogen bonds with C621 and Y658 residues and a unique halogen bond between the fluorine of DNFB and Y684 residue (Fig. 5), consistent with the observation that a non-covalent ligand binding confers a biased agonism of TRPA1 channels (Liu et al., 2020). The non-covalent binding agonist DNFB that activates TRPA1 without causing the channel desensitization is widely used for the model establishment of persistent dermatitis (Kaplan et al., 2012), which is unlike agonist AITC that covalently binds to and induces TRPA1 channel desensitization (Dai et al., 2007; Liu et al., 2020). Other non-covalent TRPA1 agonists such as peptide scorpion toxin (WaTx) or small molecule GNE551 can activate TRPA1 in a slow kinetics fashion without inducing channel desensitization and cause persistent pain (Lin King et al., 2019; Liu et al., 2020). Non-covalent agents are non-reactive to cytosolic abundant nucleophiles (such as glutathione) and are expected to sustain their concentration for longer time, thus leading to more persistent activation of TRPA1 (Liu et al., 2020). In contrast, covalent agents are highly reactive and are not stable in a cytosolic environment containing high concentrations of nucleophiles (Liu et al., 2020). Covalent TRPA1 agonists such as benzoquinone, JT010 and AITC, covalently binding to the cysteine residues of the electrophilic sensing domain of TRPA1, can make TRPA1 desensitization and deactivation, and only cause acute pain (Heber et al., 2019; Ibarra and Blair, 2013; Suo et al., 2020). We also made an effort in docking of DNCB, a derivative of DNFB, into the same 
electrophilic sensing pocket, which reveals a similar non-covalent binding through hydrogen bonds without the halogen bond to Y684 (data not shown) and DNCB also activates TRPA1, but the skin sensitization mediated by DNCB is much milder than that of DNFB (Hsieh et al., 1996; Tingle et al., 1990).

In summary, we identify the skin sensitizer DNFB that selectively activates TRPA1 channel through binding to the channel irritant sensing domain. DNFB can serve as molecular tool for better understanding of TRPA1 pharmacology and pathology. In addition, pharmacological inhibition of TRPA1 channel may hold a promise for therapy of dermatitis.

\section{Author contributions}

Han Wu, Xiaoying Sun and KeWei Wang designed the project; Han Wu, Yaxuan Qu and Xiaoying Sun performed the project; Yaxuan $\mathrm{Qu}$ and $\mathrm{Han} \mathrm{Wu}$ analyzed the data; Han $\mathrm{Wu}$, Yaxuan Qu, Xiaoying Sun and KeWei Wang wrote the manuscript.

\section{Acknowledgements}

We are grateful to Drs. Fan Yang, Xiaowen Tang and Mr. Heng Zhang for assistance and discussion in the molecular docking. This work was supported by National Natural Science Foundation of China (81903734 and 81973299) and the Ministry of Science and Technology of China (2018ZX09711001-004-006).

\section{Conflict of interest}

The authors declare no conflicts of interest.

\section{Declaration of transparency and scientific rigour}

This declaration acknowledges that this paper adheres to the principles for transparent reporting and scientific rigour of preclinical research as stated in the BJP guidelines for Design \& Analysis and as recommended by funding agencies, publishers and other organisations engaged with supporting research.References

Atoyan, R., Shander, D., and Botchkareva, N.V. (2009). Non-neuronal expression of transient receptor potential type A1 (TRPA1) in human skin. The Journal of investigative dermatology 129 , 2312-2315.

Bandell, M., Story, G.M., Hwang, S.W., Viswanath, V., Eid, S.R., Petrus, M.J., Earley, T.J., and Patapoutian, A. (2004). Noxious cold ion channel TRPA1 is activated by pungent compounds and bradykinin. Neuron 41, 849-857.

Bertin, S., Aoki-Nonaka, Y., Lee, J., de Jong, P.R., Kim, P., Han, T., Yu, T., To, K., Takahashi, N., Boland, B.S., et al. (2017). The TRPA1 ion channel is expressed in CD4+T cells and restrains T-cell-mediated colitis through inhibition of TRPV1. Gut $66,1584-1596$.

Bhol, K.C., and Schechter, P.J. (2005). Topical nanocrystalline silver cream suppresses inflammatory cytokines and induces apoptosis of inflammatory cells in a murine model of allergic contact dermatitis. The British journal of dermatology 152, 1235-1242.

Cao, E., Liao, M., Cheng, Y., and Julius, D. (2013). TRPV1 structures in distinct conformations reveal activation mechanisms. Nature 504, 113-118.

Cavanaugh, E.J., Simkin, D., and Kim, D. (2008). Activation of transient receptor potential A1 channels by mustard oil, tetrahydrocannabinol and $\mathrm{Ca} 2+$ reveals different functional channel states. Neuroscience 154 , 1467-1476.

Dai, Y., Wang, S., Tominaga, M., Yamamoto, S., Fukuoka, T., Higashi, T., Kobayashi, K., Obata, K., Yamanaka, H., and Noguchi, K. (2007). Sensitization of TRPA1 by PAR2 contributes to the sensation of inflammatory pain. Journal of Clinical Investigation 117, 1979-1987.

Deng, Z.Q., Paknejad, N., Maksaev, G., Sala-Rabanal, M., Nichols, C.G., Hite, R.K., and Yuan, P. (2018). Cryo-EM and X-ray structures of TRPV4 reveal insight into ion permeation and gating mechanisms. Nature structural \& molecular biology 25, 252. 
Engel, M.A., Leffler, A., Niedermirtl, F., Babes, A., Zimmermann, K., Filipovic, M.R., Izydorczyk, I., Eberhardt, M., Kichko, T.I., Mueller-Tribbensee, S.M., et al. (2011). TRPA1 and substance P mediate colitis in mice. Gastroenterology $141,1346-1358$.

Garcia-Perez, A. (1978). Occupational dermatitis from DNFB with cross sensitivity to DNCB. Contact Dermatitis 4 , 125-127.

Heber, S., Gold-Binder, M., Ciotu, C.I., Witek, M., Ninidze, N., Kress, H.G., and Fischer, M.J.M. (2019). A Human TRPA1-Specific Pain Model. The Journal of neuroscience : the official journal of the Society for Neuroscience $39,3845-3855$.

Hinman, A., Chuang, H.H., Bautista, D.M., and Julius, D. (2006). TRP channel activation by reversible covalent modification. Proceedings of the National Academy of Sciences of the United States of America103 , 19564-19568.

Hsieh, G.C., Kolano, R.M., Andrews, W., Fey, T.A., Collins, K.A., Gauvin, D., Kawai, M., Mollison, K.W., and Luly, J.R. (1996). Immunosuppressant effects on improved guinea pig contact hypersensitivity $(\mathrm{CH})$ model: Induction with DNFB and elicitation with DNCB. Faseb Journal $10,1284-1284$.

Ibarra, Y., and Blair, N.T. (2013). Benzoquinone Reveals a Cysteine-Dependent Desensitization Mechanism of TRPA1. Molecular pharmacology 83 , 1120-1132.

Jordt, S.E., Bautista, D.M., Chuang, H.H., McKemy, D.D., Zygmunt, P.M., Hogestatt, E.D., Meng, I.D., and Julius, D. (2004). Mustard oils and cannabinoids excite sensory nerve fibres through the TRP channel ANKTM1. Nature 427, 260-265.

Kaplan, D.H., Igyarto, B.Z., and Gaspari, A.A. (2012). Early immune events in the induction of allergic contact dermatitis. Nat Rev Immunol12, 114-124.

Landsteiner, K., and Chase, M.W. (1941). Studies on the Sensitization of Animals with Simple Chemical Compounds : Ix. Skin Sensitization Induced by Injection of Conjugates. J Exp Med 73 , 431-438.

Li, M.W., Fan, X.S., Yue, Q.F., Hu, F.Y., Zhang, Y.M., and Zhu, C. (2020). The neuro-immune interaction in airway inflammation through TRPA1 expression in CD4+T cells of asthmatic mice. International Immunopharmacology 86 .

Liao, M., Cao, E., Julius, D., and Cheng, Y. (2013). Structure of the TRPV1 ion channel determined by electron cryo-microscopy. Nature504, 107-112.

Lin King, J.V., Emrick, J.J., Kelly, M.J.S., Herzig, V., King, G.F., Medzihradszky, K.F., and Julius, D. (2019). A Cell-Penetrating Scorpion Toxin Enables Mode-Specific Modulation of TRPA1 and Pain. Cell178 , 1362-1374 e1316.

Liu, C., Reese, R., Vu, S., Rouge, L., Shields, S.D., Kakiuchi-Kiyota, S., Chen, H., Johnson, K., Shi, Y.P., Chernov-Rogan, T., et al.(2020). A Non-covalent Ligand Reveals Biased Agonism of the TRPA1 Ion Channel. Neuron.

Luo, J., Feng, J., Yu, G., Yang, P., Mack, M.R., Du, J., Yu, W., Qian, A., Zhang, Y., Liu, S., et al. (2018). Transient receptor potential vanilloid 4-expressing macrophages and keratinocytes contribute differentially to allergic and nonallergic chronic itch. J Allergy Clin Immunol 141 , 608-619 e607.

Macpherson, L.J., Dubin, A.E., Evans, M.J., Marr, F., Schultz, P.G., Cravatt, B.F., and Patapoutian, A. (2007). Noxious compounds activate TRPA1 ion channels through covalent modification of cysteines. Nature445, 541-545.

Mandadi, S., and Roufogalis, B.D. (2008). ThermoTRP channels in nociceptors: taking a lead from capsaicin receptor TRPV1. Curr Neuropharmacol 6 , 21-38. 
Natsuaki, M., Higasa, M., Sagami, S., and Shinka, S. (1989). IL-1 and PGE2 productions by the regional lymph node cells from DNFB-sensitized mice. J Dermatol 16 , 111-115.

Nilius, B., Owsianik, G., Voets, T., and Peters, J.A. (2007). Transient receptor potential cation channels in disease. Physiological reviews87, 165-217.

Oh, M.H., Oh, S.Y., Lu, J., Lou, H., Myers, A.C., Zhu, Z., and Zheng, T. (2013). TRPA1-dependent pruritus in IL-13-induced chronic atopic dermatitis. J Immunol 191, 5371-5382.

Paulsen, C.E., Armache, J.P., Gao, Y., Cheng, Y., and Julius, D. (2015). Structure of the TRPA1 ion channel suggests regulatory mechanisms. Nature 520, 511-517.

Ren, S., Wang, Q., Zhang, Y., Zhang, B., Zhao, C., Dong, X., Song, Y., Zhang, W., Qin, X., and Liu, M. (2019). Imiquimod enhances DNFB mediated contact hypersensitivity in mice. Int Immunopharmacol 72, 284-291.

Rijnierse, A., Koster, A.S., Nijkamp, F.P., and Kraneveld, A.D. (2006a). TNF-alpha is crucial for the development of mast cell-dependent colitis in mice. Am J Physiol-Gastr L 291 , G969-G976.

Rijnierse, A., van Zijl, K.M.F., Koster, A.S., Nijkamp, F.P., and Kraneveld, A.D. (2006b). Beneficial effect of tachykinin NK1 receptor antagonism in the development of hapten-induced colitis in mice. European Journal of Pharmacology 548, 150-157.

Saarnilehto, M., Chapman, H., Savinko, T., Lindstedt, K., Lauerma, A.I., and Koivisto, A. (2014). Contact sensitizer 2,4-dinitrochlorobenzene is a highly potent human TRPA1 agonist. Allergy 69 , 1424-1427.

Sandor, Z., Dekany, A., Kelemen, D., Bencsik, T., Papp, R., and Bartho, L. (2016). The TRPA1 Activator Allyl Isothiocyanate (AITC) Contracts Human Jejunal Muscle: Pharmacological Analysis. Basic Clin Pharmacol Toxicol 119, 341-342.

Singh, A.K., McGoldrick, L.L., and Sobolevsky, A.I. (2018). Structure and gating mechanism of the transient receptor potential channel TRPV3. Nature structural \& molecular biology 25 , 805-813.

Southall, M.D., Li, T., Gharibova, L.S., Pei, Y., Nicol, G.D., and Travers, J.B. (2003). Activation of epidermal vanilloid receptor-1 induces release of proinflammatory mediators in human keratinocytes. The Journal of pharmacology and experimental therapeutics 304, 217-222.

Story, G.M., Peier, A.M., Reeve, A.J., Eid, S.R., Mosbacher, J., Hricik, T.R., Earley, T.J., Hergarden, A.C., Andersson, D.A., Hwang, S.W., et al. (2003). ANKTM1, a TRP-like channel expressed in nociceptive neurons, is activated by cold temperatures. Cell 112, 819-829.

Sun, X., Wei, N., Sun, X., and Wang, K.J.J.o.C.P.S. (2017). Development of a cell-based HTS adaptable calcium fluorescent assay in FlexStation3 for the screening of TRPV3 inhibitors. 26 .

Suo, Y., Wang, Z., Zubcevic, L., Hsu, A.L., He, Q., Borgnia, M.J., Ji, R.R., and Lee, S.Y. (2020). Structural Insights into Electrophile Irritant Sensing by the Human TRPA1 Channel. Neuron 105 , 882-894 e885.

Tingle, M.D., Clarke, J.B., Kitteringham, N.R., and Park, B.K. (1990). Influence of glutathione conjugation on the immunogenicity of dinitrophenyl derivatives in the rat. Int Arch Allergy Appl Immunol91, 160-165.

Toth, B.I., Olah, A., Szollosi, A.G., and Biro, T. (2014). TRP channels in the skin. Br J Pharmacol 171 , 2568-2581.

Tóth., B.I., Oláh., A., Gábor., A., Szöllo"si., and Bíró., a. (2014). TRP channels in the skin. British Journal of Pharmacology 171 .

Vay, L., Gu, C., and McNaughton, P.A. (2012). The thermo-TRP ion channel family: properties and therapeutic implications. Br J Pharmacol165, 787-801. 
Yoshioka, T., Imura, K., Asakawa, M., Suzuki, M., Oshima, I., Hirasawa, T., Sakata, T., Horikawa, T., and Arimura, A. (2009). Impact of the Gly573Ser substitution in TRPV3 on the development of allergic and pruritic dermatitis in mice. The Journal of investigative dermatology 129 , 714-722.

Zeng, D., Chen, C., Zhou, W., Ma, X., Pu, X., Zeng, Y., Zhou, W., and Lv, F. (2021). TRPA1 deficiency alleviates inflammation of atopic dermatitis by reducing macrophage infiltration. Life Sci $266,118906$.

Zhao, J., Lin King, J.V., Paulsen, C.E., Cheng, Y., and Julius, D. (2020). Irritant-evoked activation and calcium modulation of the TRPA1 receptor. Nature 585, 141-145.

\section{Figure legends}

Figure 1. Induction of dorsal skin inflammation by topical applications of chemical allergen DNFB in mice. (a) Schematic drawing of experimental procedures for generation of skin inflammation model of dorsal dermatitis by topical applications of chemical DNFB. (b) Phenotypic features of dorsal skin inflammation with and without DNFB treatment from day 1 to day 6 ( $n=5$ mice in each group). (c) Representative histological examinations of dorsal skin tissue sections (6 $\mu \mathrm{m}$ thickness) in H\&E staining with and without DNFB application at day 7 ( $n=5$ mice in each group).

Figure 2. Activation of TRPA1 channels by chemical DNFB in calcium fluorescent assay. (a, b, c and d) Normalized increase of intracellular $\mathrm{Ca}^{2+}$ levels in response to different concentrations of DNFB $(3-300 \mu \mathrm{M})$ or channel agonists of AITC $(300 \mu \mathrm{M})$ for TRPA1 (a), capsaicin $(1 \mu \mathrm{M})$ for TRPV1 (b), 2-APB $(200 \mu \mathrm{M})$ for TRPV3 (c) and GSK101 $(0.1 \mu \mathrm{M})$ for TRPV4 (d) $(n=6)$.

Figure 3. Selectivity activation of TRPA1 currents by DNFB in whole-cell recordings. (a) Whole-cell TRPA1 currents was activated by $5 \mu \mathrm{M}$ DNFB (green bar) or $300 \mu \mathrm{M}$ AITC (red bar) before inhibition by TRP channel blocker $20 \mu \mathrm{M}$ RR (pink bar) $(n=5)$. (b) TRPV1 current in response to 5 $\mu \mathrm{M}$ DNFB (green bar) or $1 \mu \mathrm{M}$ TRPV1 agonist capsaicin (red bar) before inhibition by $20 \mu \mathrm{M}$ RR (pink bar). DNFB displaying no activation on TRPV1 $(n=5)$. (c) TRPV3 current evoked by $50 \mu \mathrm{M}$ TRPV3 agonist 2-APB (red bar) or $5 \mu \mathrm{M}$ DNFB (green bar), DNFB showing no activation $(n=5)$. (d) TRPV4 current was activated by $5 \mu \mathrm{M}$ DNFB (green bar) or $1 \mu \mathrm{M}$ TRPV4 agonist $0.1 \mu \mathrm{M}$ GSK101 (red bar) before inhibition by $20 \mu \mathrm{M} R R$ (pink bar), DNFB showing no activation $(n=5)$. (e) Concentration-dependent activation of TRPA1 currents of different concentrations of DNFB $(0.1 \mu \mathrm{M}$ to $100 \mu \mathrm{M})(n=8)$ (left panel) and AITC $(n=6)(3 \mu \mathrm{M}$ to $3000 \mu \mathrm{M})$ (right panel). (f) Fitting curves by Hill equation for comparing concentration-dependent activation of hTRPA1 currents by DNFB with $\mathrm{EC}_{50}$ of $2.36 \pm 0.26 \mu \mathrm{M}(n=5-8$, green) and AITC with $\mathrm{EC}_{50}$ of $17.77 \pm 5.02 \mu \mathrm{M}(n=5-8$, red $)$.

Figure 4. Increase of single-channel open probability of TRPA1 by DNFB. (a) Activity in an inside-out patches excised from TRPA1-HEK293 cells held at $+80 \mathrm{mV} .300 \mu \mathrm{M}$ AITC (red bar) and DNFB (green bar) were added as indicated. Shown are raw current trace (upper), in an expanded time scale (middle) and NPo in 500-ms bins (lower). (b) Conductance and amplitude histograms of openings recorded at $+80 \mathrm{mV}$ were fitted with Gaussians functions (basal-black line, AITC-red line, DNFB-green line). (c) Summary for calculated mean of $\mathrm{P}_{\text {OPEN }}$ values in the presence of $300 \mu \mathrm{M}$ DNFB and AITC $(n=6-14)$. Data are shown as the mean \pm SEM. ${ }^{*} P<0.05,{ }^{* * *} P<0.001,{ }^{* * * *} P<0.0001$, by one-way analysis of variance.

Figure 5. The residues Cys621, Glu625 and Tyr 658 in the channel coupling domain critical for DNFB binding. (a) Representative bound conformations of DNFB confined to the pocket consisting of three short helixes (H2, H4 and H5) in the coupling domain in the side view (left) and the top-down view (right). (b) Local view of a DNFB and a TRPA1 subunit interactions from hydrogen bonds (green), $\pi$-Alkyl (pink) and $\pi$-sulfur (black) are shown as dotted lines. Halogen bond is circled in red. (c-g) Representative current traces of wild type mouse TRPA1 (c), C621G (d), E625A (e), Y658A (f) and E681A (g) mutants expressed in HEK293 cells in responses to DNFB and AITC. (h) Summary for normalized WT TRPA1 or mutant channel current activation by $10 \mu \mathrm{M}$ DNFB and $300 \mu \mathrm{M}$ AITC, shown by ratio of DNFB current to 
AITC current $(n=5)$. Data are shown as the mean \pm SEM. ${ }^{*} P<0.05,{ }^{* * *} P<0.001$, by unpaired $t$-test.

Figure 1

(a)

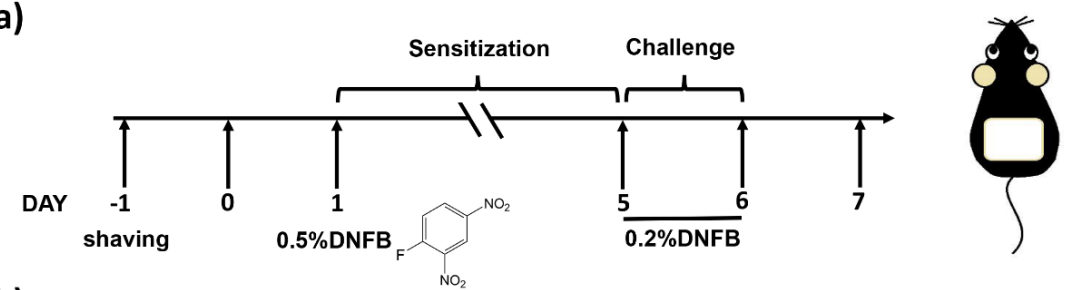

(b)

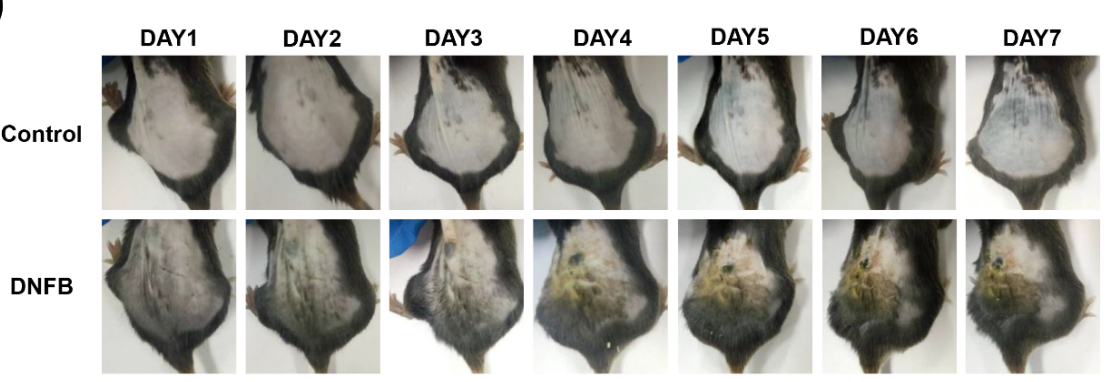

(c)
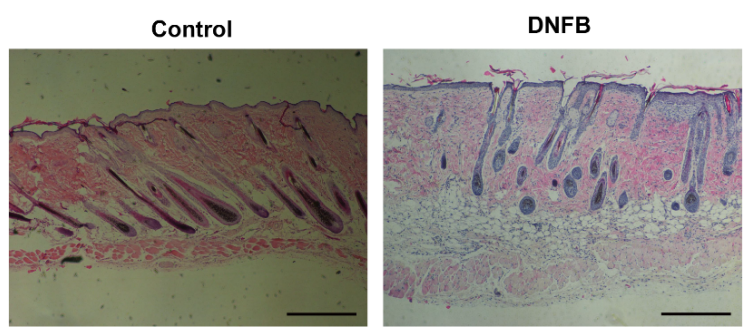
Figure 2

(a)

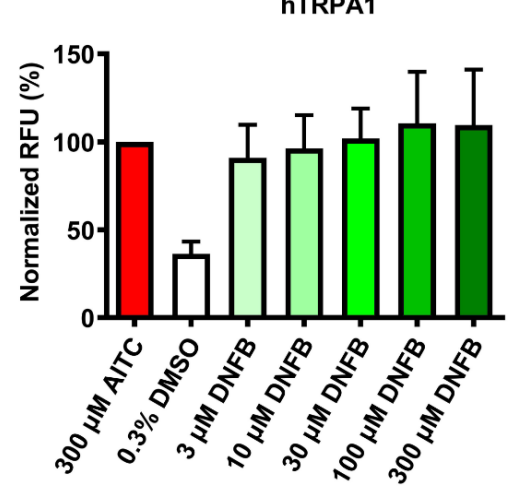

(c)

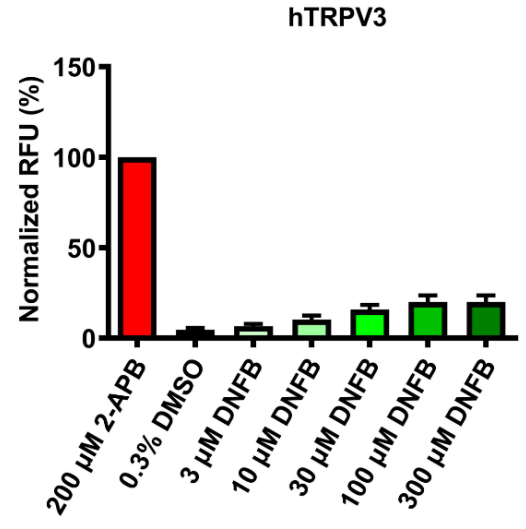

(b)

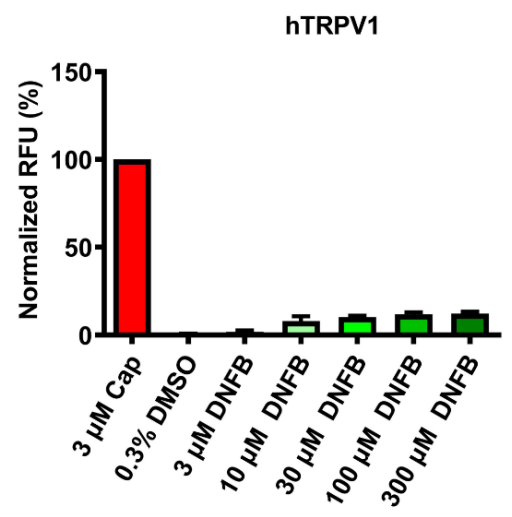

(d)

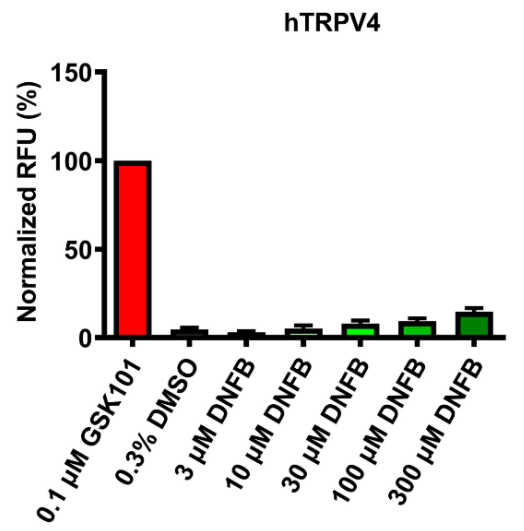


Figure 3

(a)

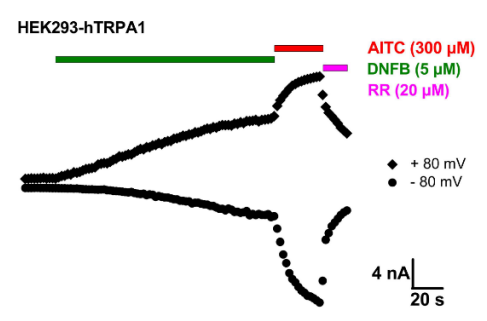

(c)

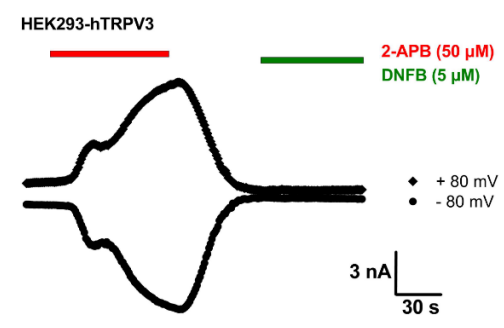

(e)

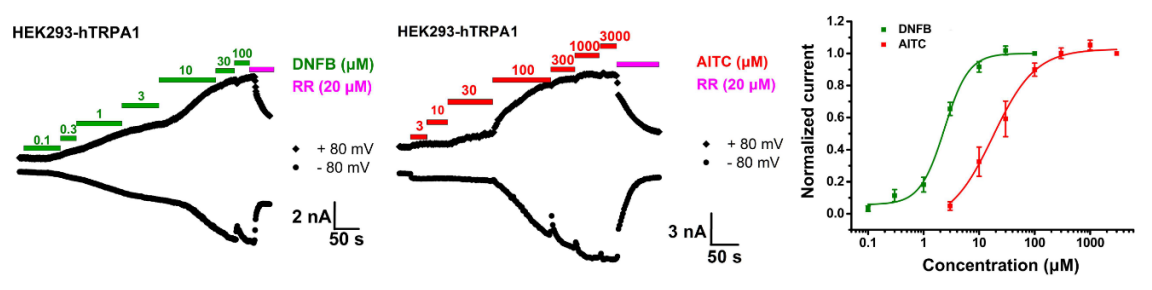

(b)

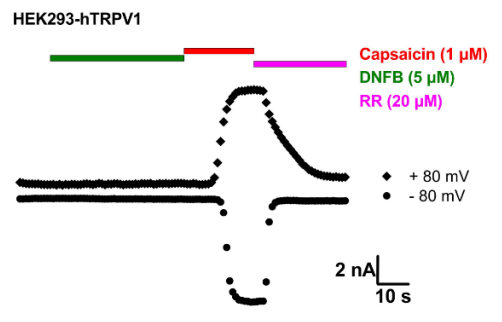

(d)

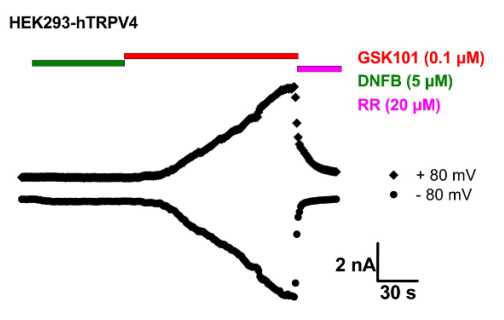


Figure 4

(a)
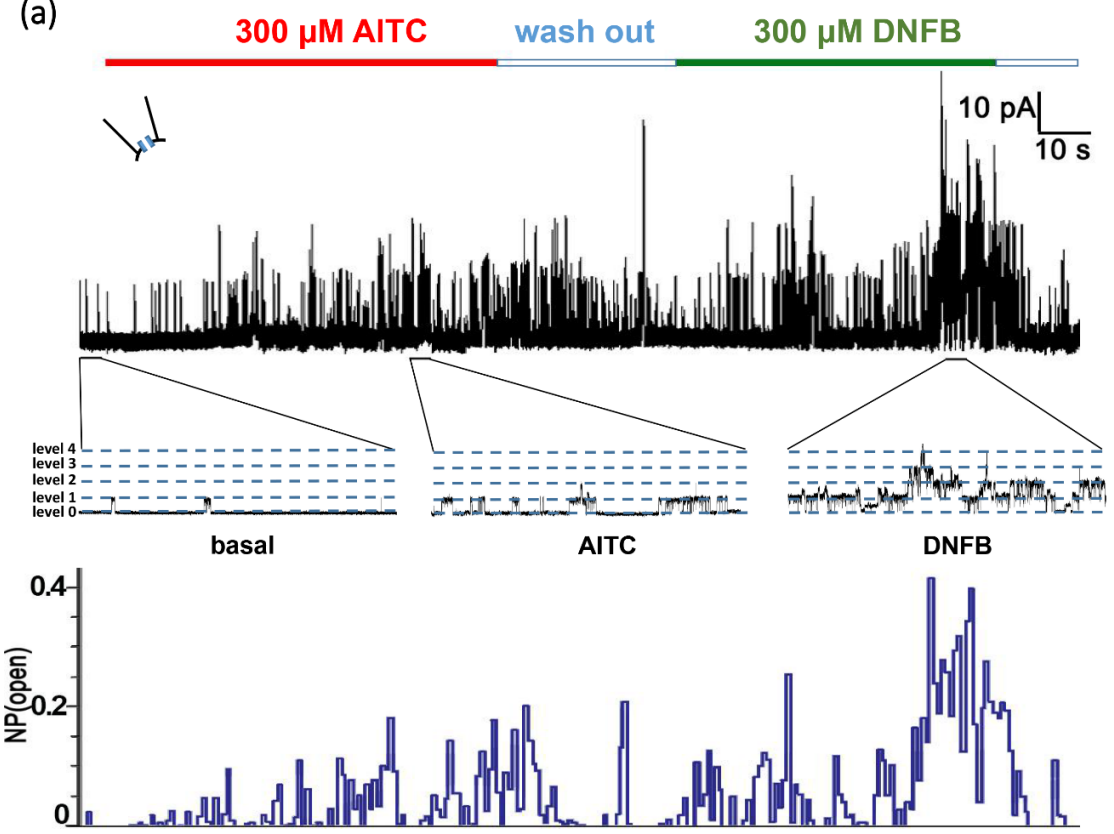

(b)
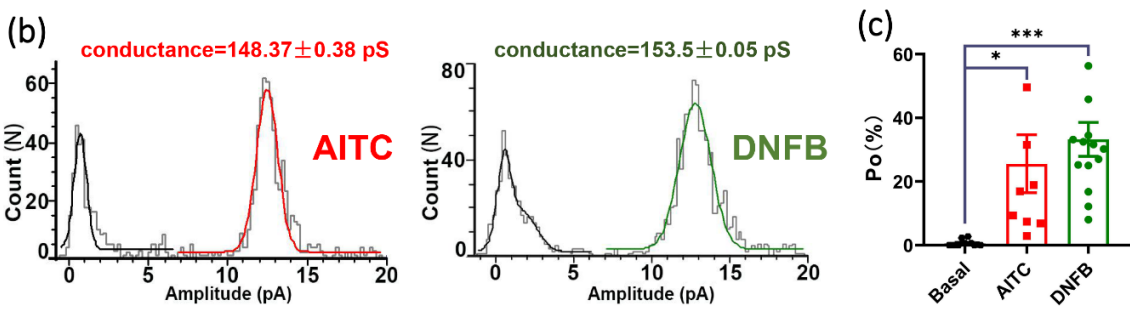
Figure 5

(a)

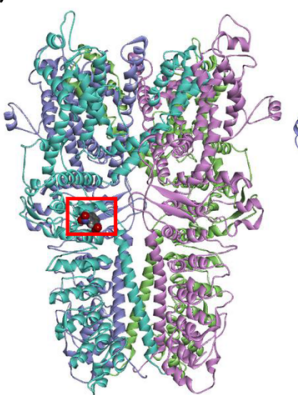

(c)

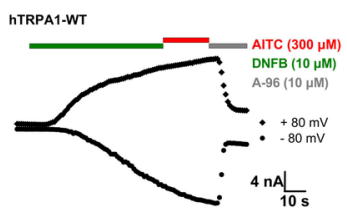

(f)

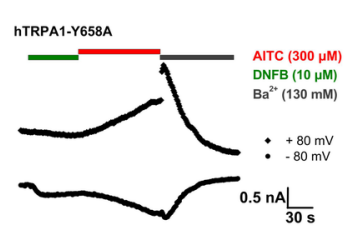

(b)

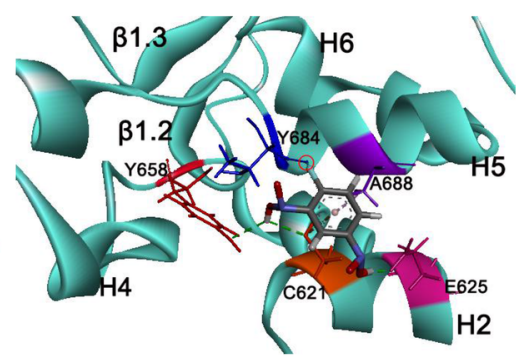

(e)

(d)

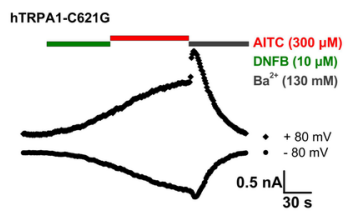

hTRPA1-E625A

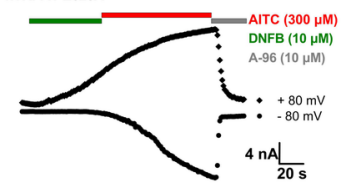

(h)

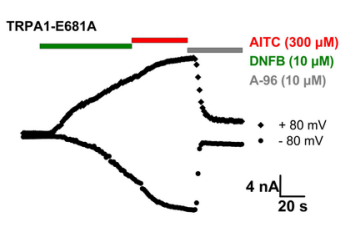

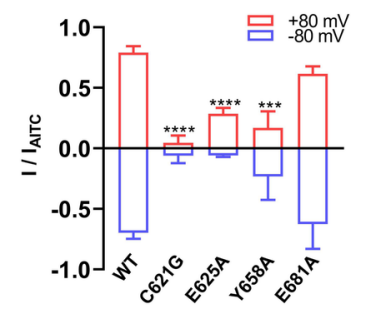


Figure 1

(a)

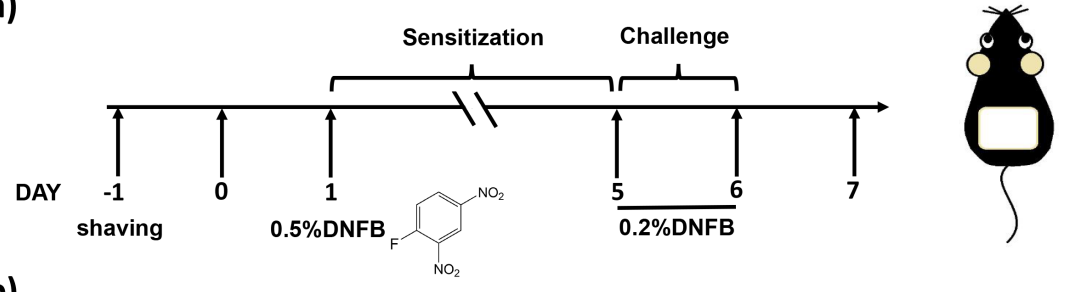

(b)

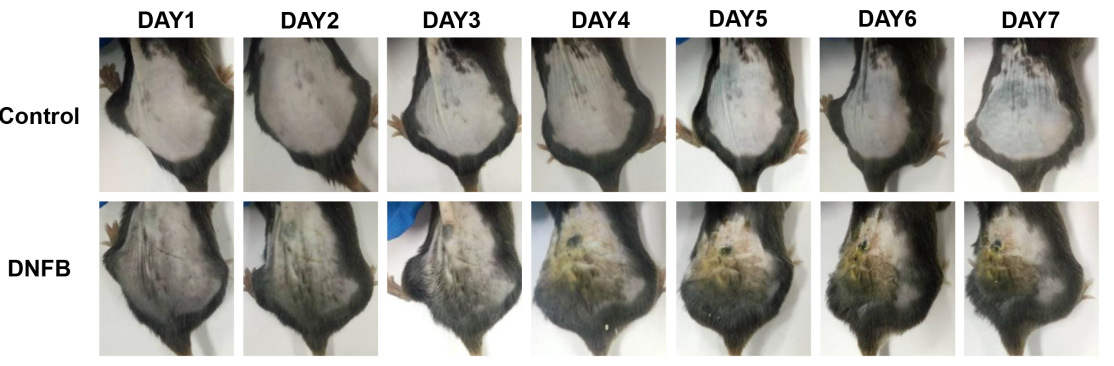

(c)
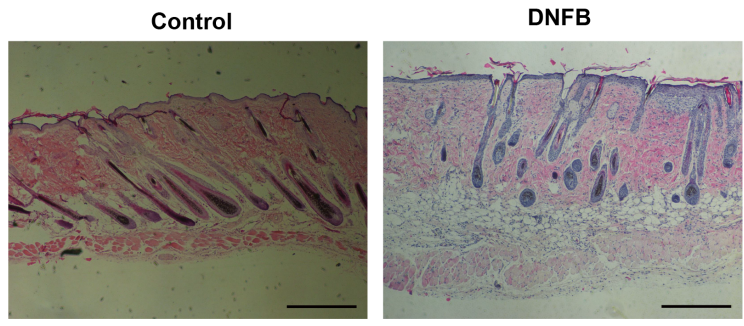
Figure 2

(a)

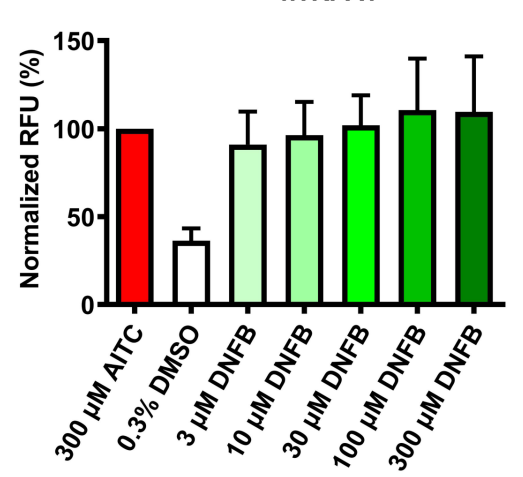

(c)

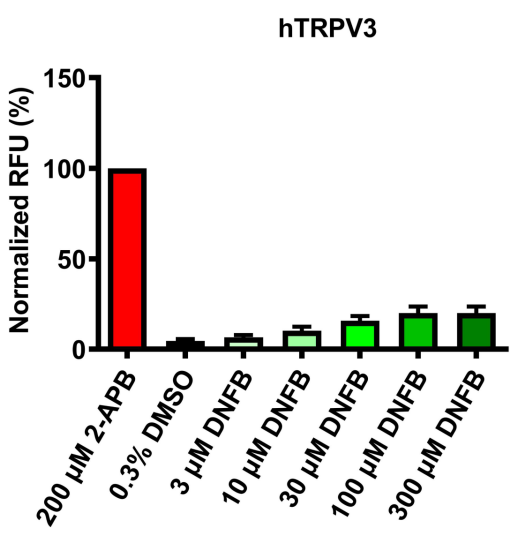

(b)

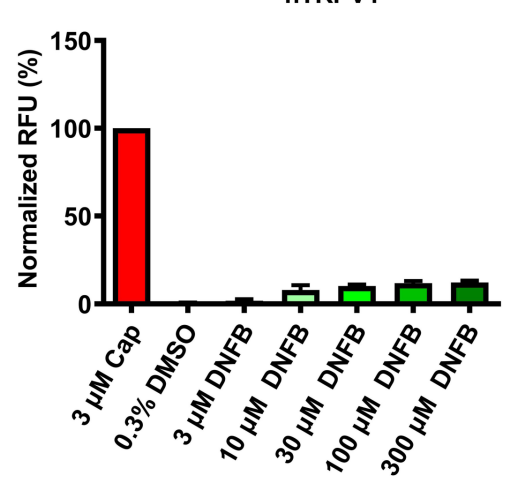

(d)

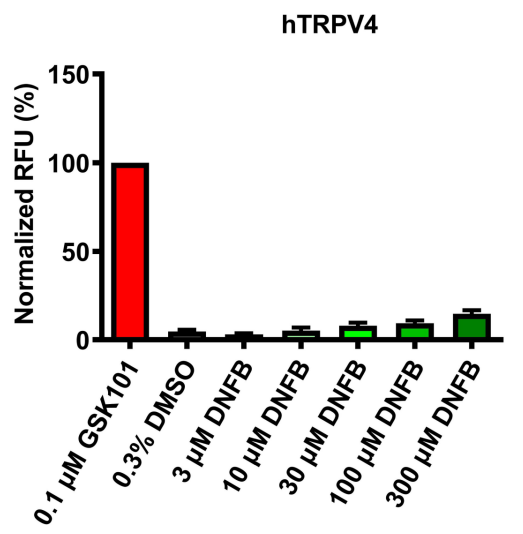


Figure 3

(a)

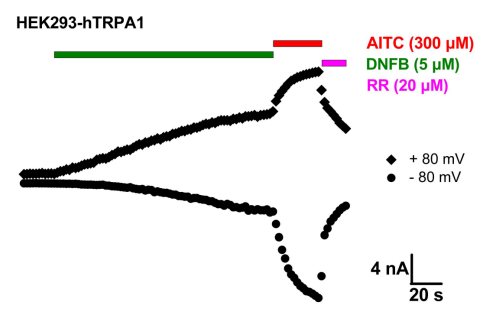

(c)

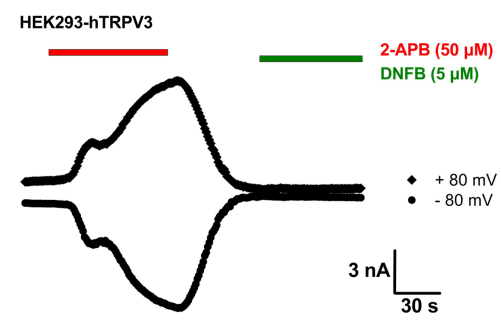

(b)

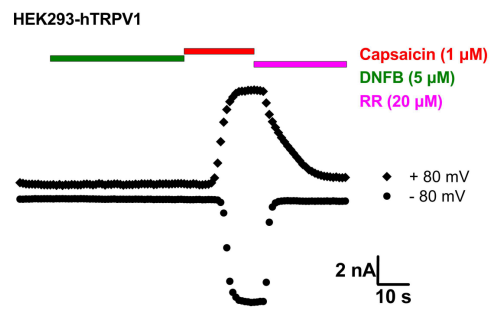

(d)

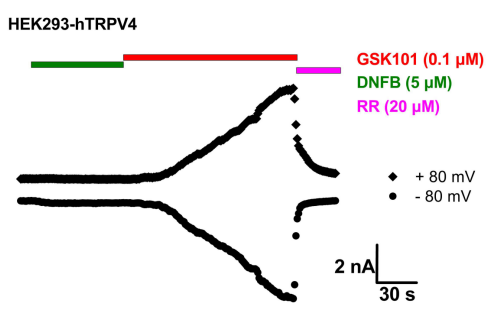

(e)

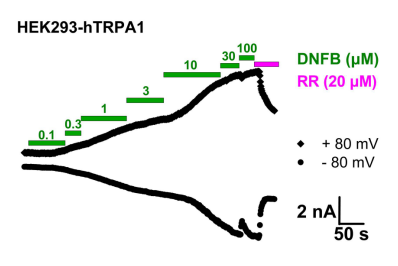

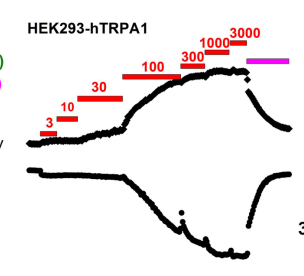

(f)

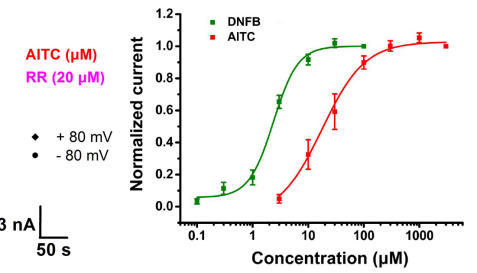


Figure 4

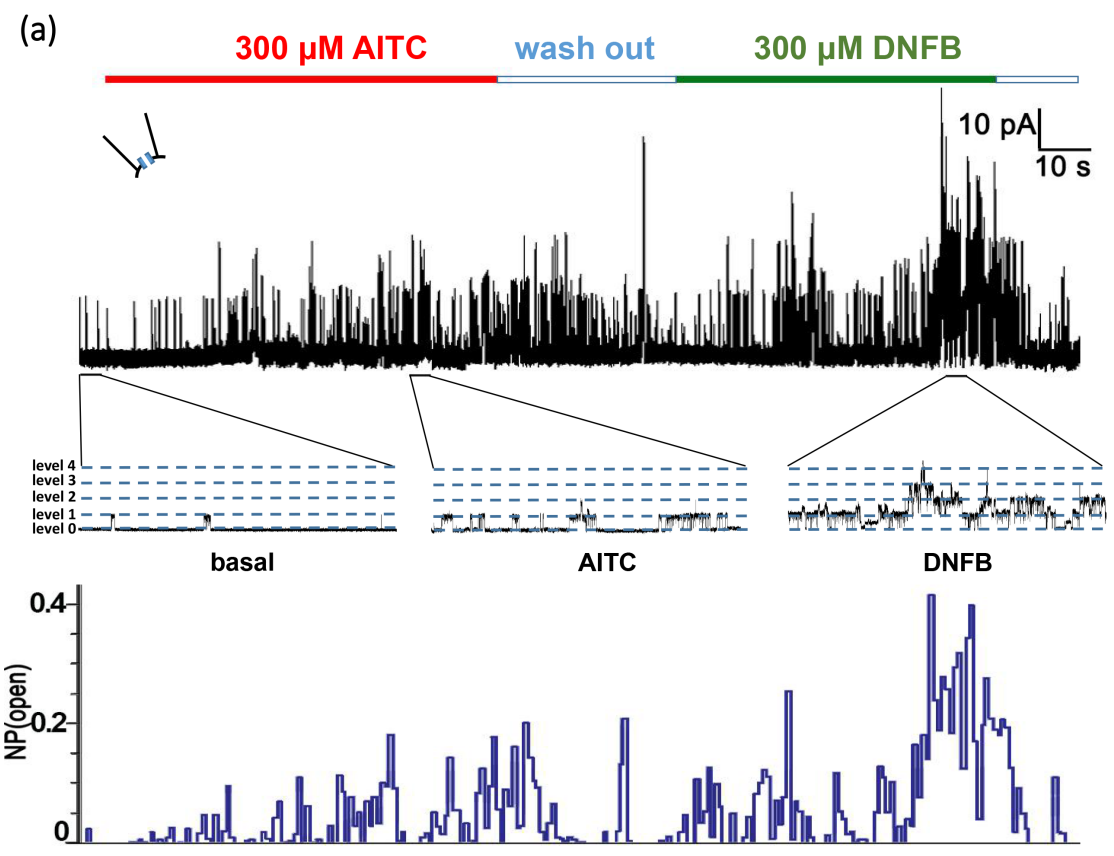

(b)
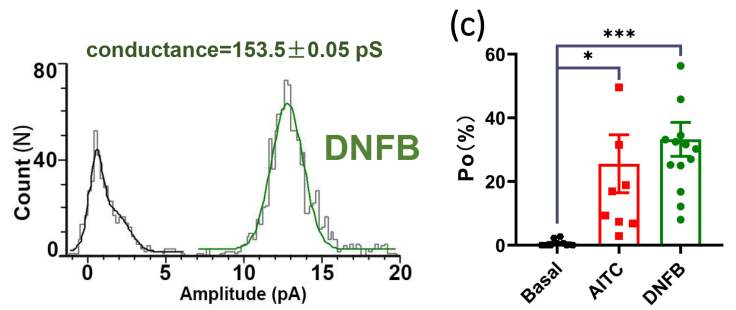
Figure 5

(a)

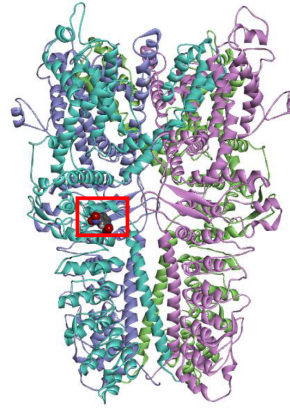

(c)

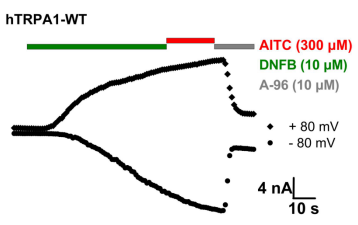

(f)

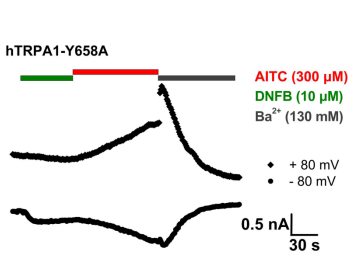

(b)

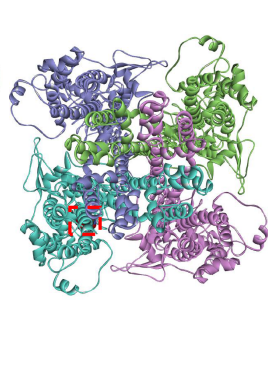

(d)

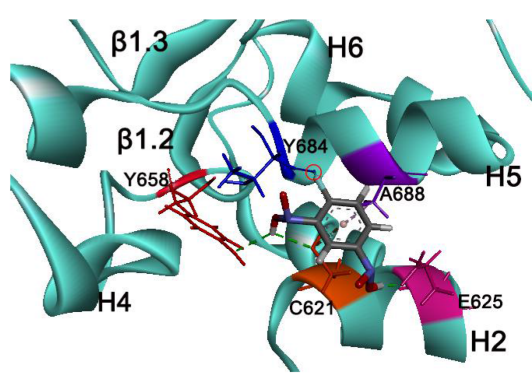

(e)

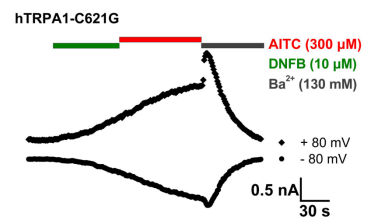

hTRPA1-E625A

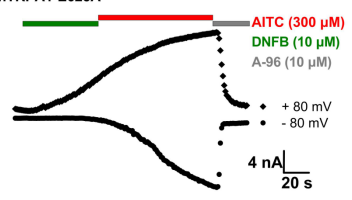

(g)

(h)

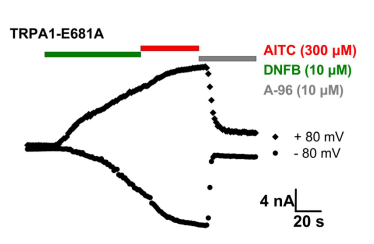

